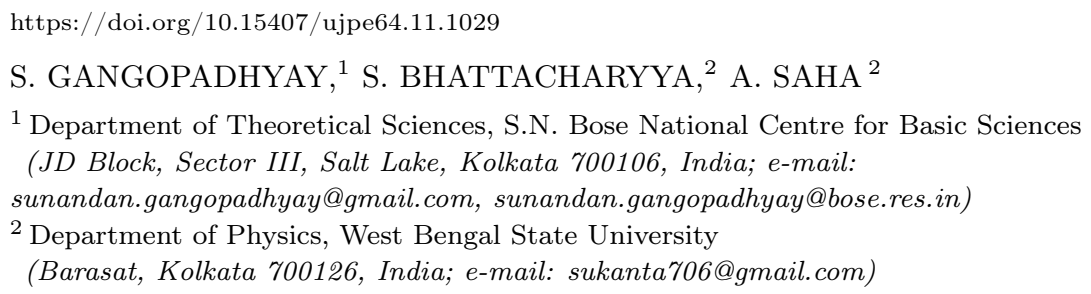

\title{
SIGNATURES OF NONCOMMUTATIVITY IN BAR DETECTORS OF GRAVITATIONAL WAVES ${ }^{1}$
}

\begin{abstract}
The comparison between the noncommutative length scale $\sqrt{\theta}$ and the length variation $\delta L=$ $=h L$, detected in the $G W$ detectors, indicates that there is a strong possibility to detect the noncommutative structure of space in the $G W$ detector setup. Therefore, we explore how the response of a bar detector gets affected due to the presence of a noncommutative structure of space keeping terms up to the second order in a gravitational wave perturbation $(h)$ in the Hamiltonian. Interestingly, the second-order term in $h$ shows a transition between the ground state and one of the perturbed second excited states that was absent, when the calculation was restricted only to the first order in $h$.
\end{abstract}

Ke y words: gravitational waves, noncommutative (NC), NC quantum field theory, NC quantum mechanics.

\section{Introduction}

The existence of an uncertainty in the spatial coordinates $[1,2]$ due to a sharp localization of events in space at the quantum level is strongly supported by various gedanken experiments. The standard way to impose this uncertainty is to postulate the noncommutative (NC) Heisenberg algebra [3]

$\left[\hat{x}_{i}, \hat{x}_{j}\right]=i \theta \epsilon_{i j}, \quad\left[\hat{x}_{i}, \hat{p}_{j}\right]=i \hbar \delta_{i j}$,

$\left[\hat{p}_{i}, \hat{p}_{j}\right]=0 ; \quad i, j=1,2$,

where $\theta$ is the NC parameter, and $\epsilon_{i j}$ is an antisymmetric tensor with $\epsilon_{12}=1$. With this granular structure of space, the NC quantum field theory (NCQFT) $[3,4], \mathrm{NC}$ quantum mechanics (NCQM) [5-11], and NC gravity [12-14] have been constructed. A part of the endeavor has also been spent in finding the order of magnitude of the $\mathrm{NC}$ parameter and exploring its connection with observations [15-21]. Studies in NCQM suggest that the NC parameter associated with different particles may not be the same [19,20], and this upperbound on the length scale could be as high as $\sqrt{\theta} \sim 10^{-20} \mathrm{~m} \div 10^{-17} \mathrm{~m}[21]$.

Length scales of this order appear in a completely different context. The direct detection of the gravita-

(C) S. GANGOPADHYAY, S. BHATTACHARYYA,

A. SAHA, 2019

ISSN 2071-0194. Ukr. J. Phys. 2019. Vol. 64, No. 11 tional waves (GWs) $[22,23]$ by the advanced LIGO detector [24] has opened a new window to observe variations in length-scales that has never been probed before. Among the currently operating GW detectors [25-30] (LIGO [31], VIRGO [32], GEO [33], and TAMA [34]), where the interferometric techniques are being used, one can detect a length-variation of the order of $\frac{\delta L}{L} \sim 10^{-23}$. Interestingly, the upper bound on the spatial NC parameter also has a length scale of this order $\left(\sqrt{\theta} \approx 10^{-20} \mathrm{~m}\right)$.

This motivates us to anticipate that a good possibility of detecting the NC structure of space would be in the GW detection experiments. It turns out that the response of a resonant bar detector to GW can be quantum mechanically described as the GWharmonic oscillator (GW-HO) [35] interaction.

To predict the possible presence of an NC structure of space in GW detection scenarios, we had studied various aspects of the GW-HO interaction in the NCQM framework in [36-42]. In those studies, we have worked out the response of a system which indicates that the spatial noncommutativity introduces a characteristic shift in the frequency, where

1 This work is based on the results presented at the XI BolyaiGauss-Lobachevskii (BGL-2019) Conference: Non-Euclidean, Noncommutative Geometry and Quantum Physics. 
the $\mathrm{HO}$ will resonate with the GW. In [42], we worked out the complete perturbative calculation including both the time-independent and time-dependent interacting Hamiltonians to obtain transition probabilities among the shifted energy levels for a generic GW wave-form up to the first order in $h$. Here, we investigate how the transitions get affected due to the second-order terms in $h$. Interestingly, we observe that there is no contribution from the $\mathcal{O}\left(h^{2}\right)$ terms in the transition probabilities calculated earlier in [42]. It only gives rise to another transition between the ground state to one of the perturbed second exited states of the HO. This transition was absent at the first order in $h$ in our earlier study.

\section{NC HO-GW Interaction}

In a resonant bar detector, the phonon mode excitations [35] interacting with the incoming GW behave themselves like a quantum mechanical forced HO. We therefore set out to analyze a quantum mechanical forced $\mathrm{HO}$ to explore the theory of interaction of GW with a resonant bar detector. Here, we consider the effect of the GW to be constrained in the $2 \mathrm{D}$ plane (taken in the $x-y$ plane) perpendicular to the GW propagation direction.

To construct the theoretical framework of our model, we write the geodesic deviation equation for a $2 \mathrm{D} \mathrm{HO}$ with mass $m$ and intrinsic frequency $\varpi$ in a proper detector frame as

$m \ddot{x}^{j}=-m R^{j}{ }_{0 k 0} x^{k}-m \varpi^{2} x^{j}$,

where $R_{0 k 0}^{j}=-\frac{d \Gamma_{0 k}^{j}}{d t}=-\ddot{h}_{j k} / 2, R_{0 k 0}^{j}$ denotes the components of the curvature tensor in terms of the metric perturbation $h_{\mu \nu}$ as $g_{\mu \nu}=\eta_{\mu \nu}+h_{\mu \nu}$; and $\left|h_{\mu \nu}\right| \ll 1$ on the flat Minkowski background $\eta_{\mu \nu}$.

It is to be noted that, here, the transverse-traceless (TT) gauge has been used to remove the unphysical degrees of freedom. The TT gauge choice gives only two physical degrees of freedom, namely, the $\times$ and + polarizations of the GW. A convenient form of $h_{j k}$ showing these polarizations reads

$h_{j k}(t)=2 f\left(\varepsilon_{\times} \sigma_{j k}^{1}+\varepsilon_{+} \sigma_{j k}^{3}\right)$,

where $2 f$ is the amplitude of the GW, $\sigma^{1}$ and $\sigma^{3}$ are the Pauli spin matrices, and the two possible polarization states of the $\mathrm{GW},\left(\varepsilon_{\times}, \varepsilon_{+}\right)$are restricted to follow the condition $\varepsilon_{\times}^{2}+\varepsilon_{+}^{2}=1$ for all $t$.

1030
The Lagrangian which leads to the geodesic deviation equation (2) is given by

$\mathcal{L}=\frac{1}{2} m{\dot{x_{j}}}^{2}-m \Gamma^{j}{ }_{0 k} \dot{x}_{j} x^{k}-\frac{1}{2} m \varpi^{2} x_{j}^{2}$.

This immediately leads to the Hamiltonian

$H=\frac{1}{2 m}\left(p_{j}+m \Gamma_{0 k}^{j} x^{k}\right)^{2}+\frac{1}{2} m \varpi^{2} x_{j}^{2}$.

In this paper, we set out to find the signature of the spatial noncommutativity considering the effect of second-order terms in the perturbation $h_{i j}$ appearing in our model. To carry this out, first, we elevate the phase-space variables $\left(x^{j}, p_{j}\right)$ to operators $\left(\hat{x}^{j}, \hat{p}_{j}\right)$ and impose the NC Heisenberg algebra. The NC operators are connected to the operators $X_{i}$ and $P_{i}$ satisfying the standard $(\theta=0)$ Heisenberg algebra through

$\hat{x}_{i}=X_{i}-\frac{1}{2 \hbar} \theta \epsilon_{i j} P_{j}, \quad \hat{p}_{i}=P_{i}$.

Using this connection, the Hamiltonian in Eq. (5) can be written in terms of the commutative variables up to the second order in $\Gamma$ as

$\hat{H}=\left(\frac{P_{j}^{2}}{2 m}+\frac{1}{2} m \varpi^{2} X_{j}^{2}\right)+\Gamma_{0 k}^{j} X_{j} P_{k}-$
$-\frac{m \varpi^{2}}{2 \hbar} \theta \epsilon_{j m} X^{j} P_{m}-\frac{\theta}{2 \hbar} \epsilon_{j m} P_{m} P_{k} \Gamma_{0 k}^{j}+$
$+\frac{m}{2} \Gamma_{0 k}^{j} \Gamma_{0 l}^{j} X^{k} X^{l}+\mathcal{O}\left(\theta \Gamma^{2}\right)+\mathcal{O}\left(\theta^{2}\right)$.

The above Hamiltonian in terms of the raising and lowering operators can be recast as

$\hat{H}=\hbar \varpi\left(a_{j}^{\dagger} a_{j}+1\right)-\frac{i \hbar}{4} \dot{h}_{j k}(t)\left(a_{j} a_{k}-a_{j}^{\dagger} a_{k}^{\dagger}\right)+$

$+\frac{m \varpi \theta}{8} \epsilon_{j m} \dot{h}_{j k}(t)\left(a_{m} a_{k}-a_{m} a_{k}^{\dagger}+C . C\right)+$

$+\frac{\hbar}{4 \varpi} \dot{h}_{j k}(t) \dot{h}_{j l}(t)\left(a_{k} a_{l}+a_{k} a_{l}^{\dagger}+a_{k}^{\dagger} a_{l}+a_{k}^{\dagger} a_{l}^{\dagger}\right)-$

$-\frac{i}{2} m \varpi^{2} \theta \epsilon_{j k} a_{j}^{\dagger} a_{k}$

where C.C. means complex conjugate. The raising and lowering operators in terms of the oscillator frequency $\varpi$ are given by

$X_{j}=\sqrt{\frac{\hbar}{2 m \varpi}}\left(a_{j}+a_{j}^{\dagger}\right) ; P_{j}=\sqrt{\frac{\hbar m \varpi}{2 i}}\left(a_{j}-a_{j}^{\dagger}\right)$.

ISSN 2071-0194. Ukr. J. Phys. 2019. Vol. 64, No. 11 
We now write Hamiltonian (8) into three parts as

$\hat{H}=\hat{H}_{0}+\hat{H}_{1}(t)+\hat{H}_{2}$,

$\hat{H}_{0}=\hbar \varpi\left(a_{j}^{\dagger} a_{j}+1\right)$,

$\hat{H}_{1}(t)=-\frac{i \hbar}{4} \dot{h}_{j k}(t)\left(a_{j} a_{k}-a_{j}^{\dagger} a_{k}^{\dagger}\right)+$

$+\frac{\Lambda}{4} \hbar \epsilon_{j m} \dot{h}_{j k}(t)\left(a_{m} a_{k}-a_{m} a_{k}^{\dagger}+C . C.\right)+$

$+\frac{\hbar}{4 \varpi} \dot{h}_{j k}(t) \dot{h}_{j l}(t)\left(a_{k} a_{l}+a_{k} a_{l}^{\dagger}+a_{k}^{\dagger} a_{l}+a_{k}^{\dagger} a_{l}^{\dagger}\right)$,

$\hat{H}_{2}=-i \Lambda_{\theta} \hbar \epsilon_{j k} a_{j}^{\dagger} a_{k}$,

where $\Lambda=\frac{m \varpi \theta}{2 \hbar}, \Lambda_{\theta}=\frac{m \varpi^{2} \theta}{2 \hbar}$.

The perturbed energy states incorporating the effect of the time-independent perturbation $\hat{H}_{2}$ was obtained in [42]. Those read

$$
\begin{aligned}
& \psi_{2}^{(0)}=(|2,0\rangle+|0,2\rangle), \\
& \psi_{2}^{(1)}=(|2,0\rangle-|0,2\rangle+i \sqrt{2}|1,1\rangle), \\
& \psi_{2}^{(2)}=(|2,0\rangle-|0,2\rangle-i \sqrt{2}|1,1\rangle)
\end{aligned}
$$

with the the corresponding energy eigenvalues

$E_{2}^{(0)}=3 \hbar \varpi$,

$E_{2}^{(1)}=3 \hbar \varpi\left(1+\frac{2}{3} \Lambda\right)$,

$E_{2}^{(2)}=3 \hbar \varpi\left(1-\frac{2}{3} \Lambda\right)$.

Now, we proceed to compute the transition probabilities between the ground state and the perturbed nondegenerate second excited states of a $2 \mathrm{D}$ harmonic oscillator using time-dependent perturbation theory incorporating the second order correction in $h$. The probability amplitude of the transition from the initial state $|i\rangle$ to a final state $|f\rangle,(i \neq f)$, due to a perturbation $\hat{V}(t)$, to the lowest order of approximation in time-dependent perturbation theory is given by [43]

$C_{i \rightarrow f}(t \rightarrow \infty)=-\frac{i}{\hbar} \int_{-\infty}^{t \rightarrow+\infty} d t^{\prime} e^{\frac{i}{\hbar}\left(E_{f}-E_{i}\right) t^{\prime}} \times$

$\times\left\langle\Phi_{f}\left|\hat{V}\left(t^{\prime}\right)\right| \Phi_{i}\right\rangle$.

Using the above result, we observe that the probability of transition survives only between the ground state $|0,0\rangle$ and the perturbed second excited states given by Eq. (11), where $\hat{V}\left(t^{\prime}\right)$ is given by

$\hat{V}\left(t^{\prime}\right)=-\frac{i \hbar}{4} \dot{h}_{j k}\left(t^{\prime}\right) t\left(a_{j} a_{k}-a_{j}^{\dagger} a_{k}^{\dagger}\right)+$

ISSN 2071-0194. Ukr. J. Phys. 2019. Vol. 64, No. 11
$+\frac{\Lambda}{4} \hbar \epsilon_{j m} \dot{h}_{j k}\left(t^{\prime}\right)\left(a_{m} a_{k}-a_{m} a_{k}^{\dagger}+C . C.\right)+$

$+\frac{\hbar}{4 \varpi} \dot{h}_{j k}\left(t^{\prime}\right) \dot{h}_{j l}\left(t^{\prime}\right)\left(a_{k} a_{l}+a_{k} a_{l}^{\dagger}+a_{k}^{\dagger} a_{l}+a_{k}^{\dagger} a_{l}^{\dagger}\right)$.

Expanding out the above interaction term for $j, k=$ $=1,2$, we obtain the transition amplitude between the ground state $|0,0\rangle$ and the perturbed second excited states to be

$C_{0 \rightarrow 2^{(0)}}=-\frac{i \varpi}{\hbar \sqrt{2}} \int_{-\infty}^{+\infty} d t e^{2 i \varpi t} \hbar\left(\dot{h}_{11}^{2}+\dot{h}_{12}^{2}\right)$,
$C_{0 \rightarrow 2^{(1)}}=-\frac{i}{\hbar} \int_{-\infty}^{+\infty} d t e^{2 i \varpi(1+\Lambda) t} \hbar\left[i A(\Lambda) \dot{h}_{11}(t)-\right.$

$\left.-B(\Lambda) \dot{h}_{12}(t)\right]$

$C_{0 \rightarrow 2^{(2)}}=-\frac{i}{\hbar} \int_{-\infty}^{+\infty} d t e^{2 i \varpi(1-\Lambda) t} \hbar\left[i C(\Lambda) \dot{h}_{11}(t)-\right.$

$\left.-D(\Lambda) \dot{h}_{12}(t)\right]$

where

$A(\Lambda)=\frac{1}{\sqrt{2}}(1+\Lambda), \quad B(\Lambda)=\frac{1}{\sqrt{2}}\left(\sqrt{\frac{3}{2}} \Lambda+1\right)$,
$C(\Lambda)=\frac{1}{\sqrt{2}}(1-\Lambda), \quad D(\Lambda)=\frac{1}{\sqrt{2}}\left(\sqrt{\frac{3}{2}} \Lambda-1\right)$.

Equation (15) is the main working formula in this paper. With this general formula (15), we shall compute the corresponding transition probabilities from the relation

$P_{0 \rightarrow 2}=\left|C_{0 \rightarrow 2}\right|^{2}$.

At this stage, we would like to draw attention to some interesting features about the second-order terms in $h$ on the results obtained in [42]. The above transition amplitudes (15) show that $C_{0 \rightarrow 2^{(1)}}$ and $C_{0 \rightarrow 2^{(2)}}$ contain no contribution from the second-order term in $h$. The second-order contribution in $h$ generates an additional transition between the ground state and the perturbed second excited state $\psi_{2}^{(0)}$, without altering the results for the transitions between the ground state and $\psi_{2}^{(1)}$ or $\psi_{2}^{(2)}$.

\section{Transition Probabilities for Different Types of Gravitational Waves}

In this section, we calculate the transition probabilities for different templates of gravitational wave-

1031 
forms that are likely to be generated in runaway astronomical events. First, we discuss the simplest scenario of a periodic $\mathrm{GW}$ with linear polarization. It has the form

$h_{j k}(t)=2 f_{0} \cos \Omega t\left(\varepsilon_{\times} \sigma_{j k}^{1}+\varepsilon_{+} \sigma_{j k}^{3}\right)$,

where the amplitude varies sinusoidally with a single frequency $\Omega$. The transition probabilities in this case turn out to be

$$
\begin{aligned}
& P_{0 \rightarrow 2^{(0)}}=32 \pi^{2} \varpi^{2} f_{0}^{4} \Omega^{4}\left(\varepsilon_{+}^{2}+\varepsilon_{\times}^{2}\right)^{2}[\delta(2 \varpi-2 \Omega)]^{2}, \\
& P_{0 \rightarrow 2^{(1)}}=\left(\pi f_{0} \Omega\right)^{2}\left[A(\Lambda)^{2} \varepsilon_{+}^{2}+B(\Lambda)^{2} \varepsilon_{\times}^{2}\right] \times \\
& \times\left[\delta\left(2 \varpi_{+}-\Omega\right)\right]^{2}, \\
& P_{0 \rightarrow 2^{(2)}}=\left(\pi f_{0} \Omega\right)^{2}\left[C(\Lambda)^{2} \varepsilon_{+}^{2}+D(\Lambda)^{2} \varepsilon_{\times}^{2}\right] \times \\
& \times\left[\delta\left(2 \varpi_{-}-\Omega\right)\right]^{2},
\end{aligned}
$$

where $\varpi_{+}=\varpi(1+\Lambda)$ and $\varpi_{-}=\varpi(1-\Lambda)$. The restriction on the physical range of frequencies $(0<\varpi<\infty)$ is imposed to drop the delta functions $\delta\left(2 \varpi_{+}+\Omega\right)$ and $\delta\left(2 \varpi_{-}+\Omega\right)$ that would appear in Eq. (19). The transition rates, therefore, take the form

$$
\begin{aligned}
& \lim _{T \rightarrow \infty} \frac{1}{T} P_{0 \rightarrow 2^{(1)}}=32 \pi^{2} \varpi^{2} f_{0}^{4} \Omega^{4}\left(\varepsilon_{+}{ }^{2}+\varepsilon_{\times}{ }^{2}\right)^{2} \times \\
& \times \delta(2 \varpi-2 \Omega), \\
& \lim _{T \rightarrow \infty} \frac{1}{T} P_{0 \rightarrow 2^{(1)}}=\left(\pi f_{0} \Omega\right)^{2} \times \\
& \times\left[A(\Lambda)^{2} \varepsilon_{+}{ }^{2}+B(\Lambda)^{2} \varepsilon_{\times}{ }^{2}\right] \delta\left(2 \varpi_{+}-\Omega\right) \\
& \lim _{T \rightarrow \infty} \frac{1}{T} P_{0 \rightarrow 2^{(2)}}=\left(\pi f_{0} \Omega\right)^{2} \times \\
& \times\left[C(\Lambda)^{2} \varepsilon_{+}{ }^{2}+D(\Lambda)^{2} \varepsilon_{\times}{ }^{2}\right] \delta\left(2 \varpi_{-}-\Omega\right)
\end{aligned}
$$

where we have used the relation

$\delta(\varpi)=\left[\int_{-\frac{T}{2}}^{\frac{T}{2}} d t, e^{i \varpi t}\right]=T$.

Now, looking at the expressions for $A, B, C, D$ and the transition probabilities in Eqs. (19) and (20), it is easy to see that the transition rates will be peaked around the frequencies $\Omega=2 \varpi_{+}$and $\Omega=2 \varpi_{-}$with unequal strengths. Further, the transition probabilities induced by both the + and $\times$ polarizations of the GW are affected by the spatial noncommutativity. In other words, the orientations of the GW source and the detector do not play a crucial role any more to detect the spatial NC effect. In addition to these two resonant points already observed in [42], there is another resonant point at $\Omega=\varpi$ which arises due to the second-order term in $h$. It was absent in [42]. This is a crucial result in this paper. The second-order term in $h$ gives rise to the transition probability $P_{0 \rightarrow 2^{(0)}}$. This is a purely gravity induced effect. It is also obvious from the expressions for $A, B, C, D$ in Eq. (16) that both linear and quadratic terms in the dimensionless NC parameter $\Lambda$ will appear in the transition probabilities (19).

The characteristic NC parameter $\Lambda$ was estimated in [40], where the stringent upper bound $|\theta| \approx 4 \times$ $\times 10^{-40} \mathrm{~m}^{2}[16]$ for the spatial noncommutativity was used. For the reference mass and frequency, the values appropriate for fundamental phonon modes of a bar detector [39] can be used. This gives

$\Lambda=\frac{m \varpi \theta}{2 \hbar}=1.888\left(\frac{m}{10^{3} \mathrm{~kg}}\right)\left(\frac{\omega}{1 \mathrm{kHz}}\right)$.

This is an interesting result, since the estimated size of the characterestic NC parameter turns out to be of the order of unity in the case of resonant bar detectors. This gives, in turn, the estimate for the characteristic $\mathrm{NC}$ frequency to be in the $\mathrm{KHz}$ range.

From the entire discussion so far, we find a very interesting feature that both the + and $\times$ polarizations include the effects of the NC structure of space. Therefore, the linearly polarized GW from a binary system with its orbital plane lying in parallel or perpendicularly to our line of sight can also be an effective test of the noncommutative structure of space. With these observations in place, we now move on to compute the transition probabilities for a circularly polarized GW. To proceed, we take the simplest form of a periodic GW signal with circular polarization, which can be conveniently expressed as

$h_{j k}(t)=2 f_{0}\left[\varepsilon_{\times}(t) \sigma_{j k}^{1}+\varepsilon_{+}(t) \sigma_{j k}^{3}\right]$,

with $\varepsilon_{+}(t)=\cos \Omega t$ and $\varepsilon_{\times}(t)=\sin \Omega t$, and $\Omega$ is the frequency of the GW.

The transition rates in this case become

$\lim _{T \rightarrow \infty} \frac{1}{T} P_{0 \rightarrow 2^{(0)}}=32 \pi^{2} \varpi^{2} f_{0}^{4} \Omega^{2} \delta(2 \varpi)=0$,

$\lim _{T \rightarrow \infty} \frac{1}{T} P_{0 \rightarrow 2^{(1)}}=\left(\frac{f_{0} \Omega}{\hbar}\right)^{2}\left[A(\Lambda)^{2}+B(\Lambda)^{2}\right] \times$ $\times \delta\left(2 \varpi_{+}-\Omega\right)$,

$\lim _{T \rightarrow \infty} \frac{1}{T} P_{0 \rightarrow 2^{(2)}}=\left(\frac{f_{0} \Omega}{\hbar}\right)^{2}\left[C(\Lambda)^{2}+D(\Lambda)^{2}\right] \times$ $\times \delta\left(2 \varpi_{-}-\Omega\right)$.

ISSN 2071-0194. Ukr. J. Phys. 2019. Vol. 64, No. 11 
From the above results, we observe that the characteristics of the transition rates for a linearly polarized GW hold for circularly polarized GW signals, as well up to the first-order in $h$. Thus, the circularly polarized GW from a binary system can also serve as a deterministic probe for the spatial noncommutativity. However, if we consider the effect of the second order term in $h$, the transition probability $P_{0 \rightarrow 2^{(0)}}$ is absent for a circularly polarized GW, which is clearly different from that for a linearly polarized GW. This interesting result can be used to determine the type of polarization of the GW source.

Now, we proceed to investigate the status of our system interacting with aperiodic GW signals, which are basically generated from GW bursts. GW bursts can be modelled by taking a simple choice as

$h_{j k}(t)=2 f_{0} g(t)\left(\varepsilon_{\times} \sigma_{j k}^{1}+\varepsilon_{+} \sigma_{j k}^{3}\right)$

containing both components of the linear polarization. We further take a Gaussian form for the function $g(t)$ :

$g(t)=e^{-t^{2} / \tau_{g}^{2}}$,

with $\tau_{g} \sim \frac{1}{f_{\max }}$, where $f_{\max }$ is the maximum value of a broad range continuum spectrum of frequencies. Note that, at $t=0, g(t)$ goes to unity. Now, the Fourier decomposed modes of the GW burst can be written as

$h_{j k}(t)=\frac{f_{0}}{\pi}\left(\varepsilon_{\times} \sigma_{j k}^{1}+\varepsilon_{+} \sigma_{j k}^{3}\right) \int_{-\infty}^{+\infty} \tilde{g}(\Omega) e^{-i \Omega t} d \Omega$,

where $\tilde{g}(\Omega)=\sqrt{\pi} \tau_{g} e^{-\left(\frac{\Omega \tau_{g}}{2}\right)^{2}}$ is the amplitude of the Fourier mode at the frequency $\Omega$.

The transition probabilities induced by a GW burst can now be computed and read [42]

$$
\begin{aligned}
& P_{0 \rightarrow 2^{(0)}}=2 \varpi^{6} f_{0}^{4} \tau_{g}^{4} e^{-\tau_{g}^{2} \varpi^{2}}\left[\varepsilon_{+}^{2}+\varepsilon_{\times}^{2}\right]^{2}, \\
& P_{0 \rightarrow 2^{(1)}}=\left[4 \sqrt{\pi} f_{0} \tau_{g} \varpi_{+}\right]^{2} e^{-2 \tau_{g}^{2} \varpi_{+}^{2} \times} \\
& \times\left(A(\Lambda)^{2} \varepsilon_{+}{ }^{2}+B(\Lambda)^{2} \varepsilon_{\times}{ }^{2}\right), \\
& P_{0 \rightarrow 2^{(2)}}=\left[4 \sqrt{\pi} f_{0} \tau_{g} \varpi_{-}\right]^{2} e^{-2 \tau_{g}^{2} \varpi_{-}^{2} \times} \\
& \times\left(C(\Lambda)^{2} \varepsilon_{+}{ }^{2}+D(\Lambda)^{2} \varepsilon_{\times}{ }^{2}\right) .
\end{aligned}
$$

Lastly, we consider a modulated Gaussian function $g(t)$ of the form

$g(t)=e^{-t^{2} / \tau_{g}^{2}} \sin \Omega_{0} t$, which represents a more realistic model of the GW burst signal. The Fourier transform of this function reads

$$
\begin{aligned}
& \tilde{g}(\Omega)=2 \pi \int_{-\infty}^{+\infty} g(t) e^{i \Omega t} d \Omega= \\
& =\frac{i \sqrt{\pi} \tau_{g}}{2}\left[e^{-\left(\Omega-\Omega_{0}\right)^{2} \tau_{g}^{2} / 4}-e^{-\left(\Omega+\Omega_{0}\right)^{2} \tau_{g}^{2} / 4}\right] .
\end{aligned}
$$

From this waveform, we get the transition amplitudes to be

$$
\begin{aligned}
& C_{0 \rightarrow 2^{(1)}}=\frac{i}{2 \sqrt{2}} \varpi^{3} f_{0}^{2} \tau_{g}^{2}\left(\varepsilon_{\times}^{2}+\varepsilon_{+}^{2}\right) \times \\
& \times\left[e^{-\frac{\left(\varpi-\Omega_{0}\right)^{2} \tau_{g}^{2}}{4}}-e^{-\frac{\left(\varpi+\Omega_{0}\right)^{2} \tau_{g}^{2}}{4}}\right]^{2}, \\
& C_{0 \rightarrow 2^{(1)}}=\left[e^{-\left(2 \varpi_{+}-\Omega_{0}\right)^{2} \tau_{g}^{2} / 4}-e^{-\left(2 \varpi_{+}+\Omega_{0}\right)^{2} \tau_{g}^{2} / 4}\right] \times(30) \\
& \times\left(2 \sqrt{\pi} f_{0} \varpi_{+} \tau_{g}\right)\left(A(\Lambda) \varepsilon_{+}+B(\Lambda) \varepsilon_{\times}\right), \\
& C_{0 \rightarrow 2^{(2)}}=\left[e^{-\left(2 \varpi_{+}-\Omega_{0}\right)^{2} \tau_{g}^{2} / 4}-e^{-\left(2 \varpi_{+}+\Omega_{0}\right)^{2} \tau_{g}^{2} / 4}\right] \times \\
& \times\left(2 \sqrt{\pi} f_{0} \varpi_{+} \tau_{g}\right)\left(C(\Lambda) \varepsilon_{+}+D(\Lambda) \varepsilon_{\times}\right) .
\end{aligned}
$$

The corresponding transition probabilities are

$$
\begin{aligned}
& P_{0 \rightarrow 2^{(0)}}=\frac{1}{8} \varpi^{6} f_{0}^{4} \tau_{g}^{4}\left(\varepsilon_{\times}^{2}+\varepsilon_{+}^{2}\right)^{2} \times \\
& \times\left[e^{-\frac{\left(\varpi-\Omega_{0}\right)^{2} \tau_{g}^{2}}{4}}-e^{-\frac{\left(\varpi+\Omega_{0}\right)^{2} \tau_{g}^{2}}{4}}\right]^{4}, \\
& P_{0 \rightarrow 2^{(1)}}=\left[e^{-\left(2 \varpi_{+}-\Omega_{0}\right)^{2} \tau_{g}^{2} / 4}-e^{-\left(2 \varpi_{+}+\Omega_{0}\right)^{2} \tau_{g}^{2} / 4}\right]^{2} \times(31) \\
& \times\left(2 \sqrt{\pi} f_{0} \varpi_{+} \tau_{g}\right)^{2}\left(A(\Lambda)^{2} \varepsilon_{+}{ }^{2}+B(\Lambda)^{2} \varepsilon_{\times}{ }^{2}\right), \\
& P_{0 \rightarrow 2^{(2)}}=\left[e^{-\left(2 \varpi_{-}-\Omega_{0}\right)^{2} \tau_{g}^{2} / 4}-e^{-\left(2 \varpi_{-}+\Omega_{0}\right)^{2} \tau_{g}^{2} / 4}\right]^{2} \times \\
& \times\left(2 \sqrt{\pi} f_{0} \varpi_{-} \tau_{g}\right)^{2}\left(C(\Lambda)^{2} \varepsilon_{+}{ }^{2}+D(\Lambda)^{2} \varepsilon_{\times}{ }^{2}\right) .
\end{aligned}
$$

At a low operating frequency of the detector, the two exponential terms in the transition amplitudes are almost equal and, hence, cancel. Therefore, the transition probabilities are reduced considerably. The other extreme is when $2 \varpi_{+}-\Omega_{0}=\Delta \varpi_{+}$and $2 \varpi_{-}-\Omega_{0}=\Delta \varpi_{-}$with $\frac{\Delta \varpi_{+}}{\varpi_{+}} \ll 1$ and $\frac{\Delta \varpi_{-}}{\varpi_{-}} \ll 1$, respectively. This yields [42]

$$
\begin{aligned}
& P_{0 \rightarrow 2^{(1)}} \approx \frac{1}{8} \varpi^{6} f_{0}^{4} \tau_{g}^{4}\left(\varepsilon_{\times}^{2}+\varepsilon_{+}^{2}\right)^{2} e^{-16 \varpi^{2} \tau_{g}^{2}} \\
& P_{0 \rightarrow 2^{(1)}} \approx e^{-\left(\Delta \varpi_{+}\right)^{2} \tau_{g}^{2} / 2}\left(2 \sqrt{\pi} f_{0} \varpi_{+} \tau_{g}\right)^{2} \times \\
& \times\left(A(\Lambda)^{2} \varepsilon_{+}{ }^{2}+B(\Lambda)^{2} \varepsilon_{\times}{ }^{2}\right) \\
& P_{0 \rightarrow 2^{(2)}} \approx e^{-\left(\Delta \varpi_{-}\right)^{2} \tau_{g}^{2} / 2}\left(2 \sqrt{\pi} f_{0} \varpi_{-} \tau_{g}\right)^{2} \times \\
& \times\left(C(\Lambda)^{2} \varepsilon_{+}{ }^{2}+D(\Lambda)^{2} \varepsilon_{\times}{ }^{2}\right) .
\end{aligned}
$$

ISSN 2071-0194. Ukr. J. Phys. 2019. Vol. 64, No. 11 


\section{Conclusion}

In this paper, we have extended our earlier calculations of the probabilities of transitions between the energy levels of a harmonic oscillator induced by gravitational waves in a spatial noncommutative framework including terms up to the second order in $h$. We find that, apart from the usual transition [42], there is an additional transition between the ground state and the perturbed second excited states. The probabilities of the other transitions that were present earlier [42] remained unaffected by the inclusion of second-order terms in $h$. An interesting result that we observe in our present investigation is that the additional transition probability observed in the case of linearly polarized gravitational wave (due to the inclusion of second-order terms in $h$ in the Hamiltonian) is absent in the case of a circularly polarized gravitational wave. This result can be used, in principle, to determine the type of polarization of the gravitational wave source.

1. S. Doplicher, K. Fredenhagen, J.E. Roberts. Spacetime quantization induced by classical gravity. Phys. Lett. B 331, 39 (1994).

2. D. V. Ahluwalia. Quantum measurement, gravitation, and locality. Phys. Lett. B 339, 301 (1994).

3. M.R. Douglas, N.A. Nekrasov. Noncommutative field theory. Rev. Mod. Phys. 73, 977 (2002).

4. N. Seiberg, E. Witten. String theory and noncommutative geometry. JHEP 09, 032 (1999).

5. V.P. Nair, A.P. Polychronakos. Quantum mechanics on the noncommutative plane and sphere. Phys. Lett. B 505, 267 (2001).

6. L. Mezincescu. Star operation in quantum mechanics. [hepth/0007046].

7. B. Chakraborty, S. Gangopadhyay, A. Saha. SeibergWitten map and Galilean symmetry violation in a noncommutative planar system. Phys. Rev. D 70, 107707 (2004).

8. F.G. Scholtz, B. Chakraborty, S. Gangopadhyay, A.G. Hazra. Dual families of noncommutative quantum systems. Phys. Rev. D 71, 085005 (2005).

9. F.G. Scholtz, B. Chakraborty, S. Gangopadhyay, J. Govaerts. Interactions and non-commutativity in quantum Hall systems. J. Phys. A 38, 9849 (2005).

10. S. Gangopadhyay, F.G. Scholtz. Path-integral action of a particle in the noncommutative plane. Phys. Rev. Lett. 102, 241602 (2009).

11. S. Bhattacharyya, S. Gangopadhyay, A. Saha. Quantum mechanics of a particle in an accelerated frame and the equivalence principle. Euro. Phys. Lett. 120, 30005 (2017).

12. R.J. Szabo. Symmetry, gravity and noncommutativity. Class. Quant. Grav. 23, R199 (2006).
13. P. Mukherjee, A. Saha. Note on the noncommutative correction to gravity. Phys. Rev. D 74, 027702 (2006).

14. R. Banerjee, S. Gangopadhyay, S.K. Modak. Voros product, noncommutative Schwarzschild black hole and corrected area law. Phys. Lett. B 686, 181 (2010).

15. I. Mocioiu, M. Pospelov, R. Roiban. Low-energy limits on the antisymmetric tensor field background on the brane and on the non-commutative scale. Phys. Lett. B 489, 390 (2000).

16. S.M. Carroll, J.A. Harvey, V.A. Kostelecký, C.D. Lane, T. Okamoto. Noncommutative field theory and Lorentz violation. Phys. Rev. Lett. 87, 141601 (2001).

17. O. Bertolami, J.G. Rosa, C.M.L. de Aragao, P. Castorina, D. Zappala. Noncommutative gravitational quantum well. Phys. Rev. D 72, 025010 (2005).

18. A. Saha. Time-space non-commutativity in gravitational quantum well scenario. Eur. Phys. J. C 51, 199 (2007).

19. P.M. Ho, H.C. Kao. Noncommutative quantum mechanics from noncommutative quantum field theory. Phys. Rev. Lett. 88, 151602 (2002).

20. T.C. Adorno, D.M. Gitman, A.E. Shabad, D.V. Vassilavich. Noncommutative magnetic moment of charged particles. Phys. Rev. D 84, 085031 (2011).

21. A. Stern. Noncommutative point sources. Phys. Rev. Lett. 100, 061601 (2008).

22. B.P. Abbott et al. Observation of gravitational waves from a binary black hole merger. Phys. Rev. Lett. 116, 061102 (2016).

23. B.P. Abbott et al. GW151226: Observation of Gravitational Waves from a 22-Solar-Mass Binary Black Hole Coalescence. Phys. Rev. Lett. 116, 241103 (2016).

24. https://advancedligo.mit.edu/.

25. I. Ciufolini, R.A. Matzner. General Relativity and John Archibald Wheeler (Springer, 2010) [ISBN: 9789048137350] (online).

26. P. Astone et al. Long-term operation of the Rome Explorer cryogenic gravitational wave detector. Phys. Rev. D 47, 362 (1993).

27. E. Mauceli et al. The Allegro gravitational wave detector: Data acquisition and analysis. Phys. Rev. D 54, 1264 (1996).

28. D.G. Blair et al. High sensitivity gravitational wave antenna with parametric transducer readout. Phys. Rev. Lett. 74, 1908 (1995).

29. P. Astone et al. The gravitational wave detector NAUTILUS operating at $T=0.1$ K. Astropart. Phys. 7, 231 (1997).

30. M. Cerdonio et al. The ultracryogenic gravitationalwave detector AURIGA. Class. Quant. Grav. 14, 1491 (1997).

31. A. Abrampvici et al. LIGO: The laser interferometer gravitational-wave observatory. Science 256, 325 (1992).

32. B. Caron et al. The Virgo interferometer. Class. Quant. Grav. 14, 1461 (1997).

33. H. Lück et al. The GEO600 project. Class. Quant. Grav. 14, 1471 (1997).

ISSN 2071-0194. Ukr. J. Phys. 2019. Vol. 64, No. 11 
34. M. Ando et al. Stable operation of a 300-m laser interferometer with sufficient sensitivity to detect gravitational-wave events within our galaxy. Phys. Rev. Lett. 86, 3950 (2001).

35. M. Maggiore. Gravitational Wave. Vol I. Theory and Experiments (Oxford Univ. Press, 2008) [ISBN-13: 9780198570745].

36. A. Saha, S. Gangopadhyay. Noncommutative quantum mechanics of a test particle under linearized gravitational waves. Phys. Lett. B 681, 96 (2009).

37. A. Saha, S. Gangopadhyay, S. Saha. Noncommutative quantum mechanics of a harmonic oscillator under linearized gravitational waves. Phys. Rev. D 83, 025004 (2011).

38. S. Gangopadhyay, A. Saha, S. Saha. Trace of phasespace noncommutativity in response of a free particle to linearized gravitational waves. Mod. Phys. Lett. A 28, 1350161 (2013).

39. S. Gangopadhyay, A. Saha, S. Saha. Noncommutative quantum mechanics of simple matter systems interacting with circularly polarized gravitational waves. Gen. Rel. Grav. 47, 28 (2015).

40. A. Saha, S. Gangopadhyay. Resonant detectors of gravitational wave as a possible probe of the noncommutative structure of space. Class. Quant. Grav. 33, 205006 (2016).

41. A. Saha, S. Gangopadhyay, S. Saha. Quantum mechanical systems interacting with different polarizations of gravita- tional waves in noncommutative phase space. Phys. Rev. D 97, 044015 (2018).

42. S. Bhattacharyya, S. Gangopadhyay, A. Saha. Footprint of spatial noncommutativity in resonant detectors of gravitational wave. Class. Quant. Grav. 36, 055006 (2019).

43. K. Gottfried, T.M. Yan. Quantum Mechanics: Fundamentals (Springer, 2005) [ISBN: 978-0-387-21623-2].

Received 27.08.19

C. Гангопадхяй, C. Бхатmачарія, A. Caxa

\section{СВІДЧЕННЯ НЕКОМУТАТИВНОСТІ}

ВІД ДЕТЕКТОРІВ ГРАВІТАЦЙНИХ ХВИЛЬ

$\mathrm{P}$ е $з$ ю м е

Порівняння масштабу довжини некомутативності $\sqrt{\theta}$ з варіацією довжини $\delta L=h L$, знайдене за допомогою детекторів гравітаційних хвиль, свідчить про можливість виявити некомутативну структуру простору за допомогою таких установок. Тому ми досліджуємо, як на реакцію детектора гравітаційних хвиль впливає некомутативна структура простору, зберігаючи у гамільтоніані члени до другого порядку відносно збурення гравітаційної хвилі $(h)$. Цікаво, що член другого порядку відносно $h$ відповідає за перехід між основним станом та одним із збурених станів (другим збудженим), чого не було, коли обмежувалися лише першим порядком відносно $h$. 